\title{
On Best-Possible Obfuscation
}

\author{
Shafi Goldwasser ${ }^{1,2, \star}$ and Guy N. Rothblum ${ }^{2, \star \star}$ \\ ${ }^{1}$ Weizmann Institute of Science, Rehovot 76100, Israel \\ ${ }^{2}$ CSAIL, MIT, Cambridge MA 02139, USA \\ \{shafi, rothblum\}@theory.csail.mit.edu
}

\begin{abstract}
An obfuscator is a compiler that transforms any program (which we will view in this work as a boolean circuit) into an obfuscated program (also a circuit) that has the same input-output functionality as the original program, but is "unintelligible". Obfuscation has applications for cryptography and for software protection.

Barak et al. initiated a theoretical study of obfuscation, which focused on black-box obfuscation, where the obfuscated circuit should leak no information except for its (black-box) input-output functionality. A family of functionalities that cannot be obfuscated was demonstrated. Subsequent research has showed further negative results as well as positive results for obfuscating very specific families of circuits, all with respect to black box obfuscation.

This work is a study of a new notion of obfuscation, which we call best-possible obfuscation. Best possible obfuscation makes the relaxed requirement that the obfuscated program leaks as little information as any other program with the same functionality (and of similar size). In particular, this definition allows the program to leak non black-box information. Best-possible obfuscation guarantees that any information that is not hidden by the obfuscated program is also not hidden by any other similar-size program computing the same functionality, and thus the obfuscation is (literally) the best possible. In this work we study best-possible obfuscation and its relationship to previously studied definitions. Our main results are:
\end{abstract}

1. A separation between black-box and best-possible obfuscation. We show a natural obfuscation task that can be achieved under the best-possible definition, but cannot be achieved under the black-box definition.

2. A hardness result for best-possible obfuscation, showing that strong (information-theoretic) best-possible obfuscation implies a collapse in the polynomial hierarchy.

3. An impossibility result for efficient best-possible (and black-box) obfuscation in the presence of random oracles. This impossibility result uses a random oracle to construct hard-to-obfuscate circuits, and thus it does not imply impossibility in the standard model.

\footnotetext{
* Supported by NSF grant CNS-0430450, NSF grant CFF-0635297 and a CymermanJakubskind award.

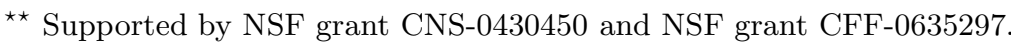




\section{Introduction}

An open question in computer security is whether computer programs can be obfuscated; whether code can be made unintelligible while preserving its functionality. This question is important as obfuscation has wide-ranging applications, both for software protection and for cryptography. Beyond its theoretical importance, the question of obfuscation is of great practical importance. Numerous ad-hoc heuristical techniques are used every day by practitioners to obfuscate their code, even though many of these techniques do not supply any provable notion of security.

A theoretical study of obfuscation was initiated by Barak, Goldreich, Impagliazzo, Rudich, Sahai, Vadhan and Yang 2. They studied several notions of obfuscation, primarily focusing on black-box obfuscation, in which an obfuscator is viewed as a compiler that, given any input program or circuit, outputs a program with the same functionality from which it is hard to find any deterministic information on the input program. Formally, black-box obfuscation requires that anything that can be efficiently computed from the obfuscated program, can also be computed efficiently from black-box (i.e. input-output) access to the program. Their main result was that this (strong) notion of obfuscation cannot always be achieved, as they were able to present an explicit family of circuits that provably cannot be black-box obfuscated.

Barak et al. 2] considered also an alternative notion of obfuscation called indistinguishability obfuscation that sidesteps the black-box paradigm. An indistinguishability obfuscator guarantees that if two circuits compute the same function, then their obfuscations are indistinguishable in probabilistic polynomial time. This definition avoids the black-box paradigm, and also avoids the impossibility results shown for the black-box obfuscation notion. Indeed, Barak et al. showed that it is simple to build inefficient indistinguishability obfuscators. One main disadvantage of indistinguishability obfuscation is that it does not give an intuitive guarantee that the circuit "hides information". This is apparent in their proposed construction of an inefficient indistinguishability obfuscator, where a small circuit is revealed which is equivalent to the original circuit. For some functionalities, this is a great deal of information to give away.

This Work. We propose a new notion of obfuscation, best-possible obfuscation, that avoids the black-box paradigm, and also gives the appealing intuitive guarantee that the obfuscated circuit leaks less information than any other circuit (of a similar size) computing the same function. This work is a study of this new notion of best-possible obfuscation.

Instead of requiring that an obfuscator strip a program of any non blackbox information, we require only that the (best-possible) obfuscated program leak as little information as possible. Namely, the obfuscated program should be "as private as" any other program computing the same functionality (and of a certain size). A best-possible obfuscator should transform any program so that anything that can be computed given access to the obfuscated program should also be computable from any other equivalent program (of some related size). 
A best-possible obfuscation may leak non black-box information (e.g. the code of a hard-to-learn function), as long as whatever it leaks is efficiently learnable from any other similar-size circuit computing the same functionality.

While this relaxed notion of obfuscation gives no absolute guarantee about what information is hidden in the obfuscated program, it does guarantee (literally) that the obfuscated code is the best possible. It is thus a meaningful notion of obfuscation, especially when we consider that programs are obfuscated every day in the real world without any provable security guarantee.

In this work we initiate a study of best-possible obfuscation. We explore its possibilities and limitations, as well as its relationship with other definitions of obfuscation that have been suggested. We formalize the best-possible requirement in Definition 5. by requiring that for every efficient learner who tries to extract information from an obfuscated circuit, there exists an efficient simulator that extracts similar information from any other circuit with the same functionality and of the same size. We consider both computationally best-possible obfuscation, where the outputs of the learner and simulator are indistinguishable with respect to efficient distinguishers, and information theoretically best-possible obfuscation (perfect or statistical), where even an unbounded distinguisher cannot tell the difference between the two. We emphasize that statistically or perfectly best-possible obfuscation refer to the distinguisher, whereas we only consider information that can be learned efficiently given the obfuscated circuit. This strengthens negative results. Our positive result on perfectly best-possible obfuscation applies also to unbounded learners.

Relationship with Previous Definitions. We study how best-possible obfuscation relates to black-box obfuscation, and present a separation between the two notions of obfuscation. The proof of this result also gives the first known separation between black-box and indistinguishability obfuscation. The separation result considers the complexity class of languages computable by polynomial sized ordered decision diagrams or POBDDs; these are log-space programs that can only read their input tape once, from left to right (see Section 3). We observe that any POBDD can be best-possible obfuscated as a POBDD (Proposition 2), whereas there are many natural functions computable by POBDDs that provably cannot be black-box obfuscated as any POBDD (Proposition 3). These two results give new possibility results (for best-possible and indistinguishability obfuscation), and simple natural impossibility results (for black-box obfuscation). Note that the impossibility result for black-box obfuscation only applies when we restrict the representation of the obfuscator's output to be a POBDD itself.

We also compare the notions of best-possible and indistinguishability obfuscation. Proposition 4 shows that any best-possible obfuscator is also an indistinguishability obfuscator. For efficient obfuscators the definitions are equivalent (Proposition 5). For inefficient obfuscation, the difference between the two definitions is sharp, as inefficient information-theoretic indistinguishability obfuscators are easy to construct (see [2]), but the existence of inefficient statistically 
best-possible obfuscators even for the class of languages recognizable by 3 -CNF circuits (a sub-class of $A C^{0}$ ) implies that the polynomial hierarchy collapses to its second level.

We believe that the equivalence of these two definitions for efficient obfuscation motivates further research on both, as the "best-possible" definition gives a strong intuitive security guarantee, and the indistinguishability definition may sometimes be technically easier to work with.

Impossibility Results. We explore the limits of best-possible obfuscation. As noted above, we begin by considering information-theoretically (statistically) best-possible obfuscation. In Theorem 1 we show that if there exist (not necessarily efficient) statistically secure best-possible obfuscators for the simple circuit family of 3-CNF circuits (a sub-class of $A C^{0}$ ), then the polynomial hierarchy collapses to its second level. Corollary 1 of this theorem states that also if there exists an efficient statistically secure indistinguishability obfuscator for the same simple circuit family, then the polynomial hierarchy collapses to its second level. This is the first impossibility result for indistinguishability obfuscation in the standard model.

We also consider best-possible obfuscation in the (programmable) random oracle model. In this model, circuits can be built using special random oracle gates that compute a completely random function. Previously, this model was considered by Lynn, Prabhakaran and Sahai [17] as a promising setting for presenting positive results for obfuscation. We show that the random oracle can also be used to prove strong negative results for obfuscation. In Theorem 2 we present a simple family of circuits with access to the random oracle, that are provably hard to best-possible obfuscate efficiently. This impossibility results extends to the black-box and indistinguishability obfuscation notions. We note that using random oracles for obfuscation was originally motivated by the hope that giving circuits access to an idealized "box" computing a random function would make it easier to obfuscate more functionalities (and eventually perhaps the properties of the "box" could be realized by a software implementation). We, on the other hand, show that the existence of such boxes (or a software implementation with the idealized properties) could actually allow the construction of circuits that are impossible to obfuscate. Although this negative result does not rule out that every circuit without random oracle gates can be best-possible obfuscated, we believe it is illuminating for two reasons. First, as a warning sign when considering obfuscation in the random oracle model, and secondly as its proof hints that achieving general purpose best-possible obfuscation in the standard model would require a significant leap (a discussion of this point appears at the end of Section 4).

\subsection{Related Work}

Negative Results. Barak et al. showed that black-box obfuscation cannot always be achieved. They showed this by presenting families of circuits that cannot be black-box obfuscated: there exists a predicate that cannot be computed from 
black-box access to a random circuit in the family, but can be computed from (non black-box access to) any circuit in the family. Thus they showed that there exist circuits that cannot be obfuscated, but it remained possible that almost any natural circuit could be obfuscated. Goldwasser and Kalai [12, showed that if the definition of obfuscation is strengthened even further with a requirement that the obfuscation leak no more information than black-box access even in the presence of auxiliary input, then a large class of more natural circuits cannot be obfuscated.

Positive Results. The functionalities for which obfuscation was ruled out in [2] and [12] are somewhat complex. An interesting open question is whether obfuscation can be achieved for simpler classes of functionalities and circuits. Lynn, Prabhakaran and Sahai 17] were the first to explicitly explore this question. They suggested working in the random oracle model and focused on obfuscating access control functionalities (note that impossibility results of [2] and [12 extend to the random oracle model). At the heart of their construction is the obfuscation of a point function. A point function $I_{p}(x)$ is defined to be 1 if $x=p$, or 0 otherwise, and they observed that in the random oracle model point functions can be obfuscated, leading to obfuscation algorithms for more complex access control functionalities. Under cryptographic assumptions, it is also known how to obfuscate point functions without a random oracle. Canetti [6] showed (implicitly) how to obfuscate point functions (even under a strong auxiliary-input definition), using a strong variant of the Decisional Diffie-Hellman assumption. Wee [21] presented a point function obfuscator based on the existence of one-way permutations that are hard to invert on a very strong sense.

Other solutions for obfuscating point functions are known if the obfuscator doesn't need to work for every point, but rather for a point selected at random from a distribution with some min-entropy. For this relaxed requirement Canetti, Micciancio and Reingold [8] presented a scheme that uses more general assumptions than those used by [6] (their solution is not, however, secure in the presence of auxiliary inputs). Dodis and Smith [9] were able to obfuscate proximity queries in this framework.

The Random Oracle Model. The random oracle model is an idealization, in which it is assumed that all parties have oracle access to a truly random function $\mathcal{R}$. The parties can access this function by querying the random oracle at different points. The Random oracle methodology is a heuristic methodology, in which the random oracle is used for building provably secure cryptographic objects, but then, to implement the cryptographic object in the real world, the random oracle is replaced by some real function with a succinct representation. This methodology was introduced by Fiat and Shamir [15], and later formalized by Bellare and Rogaway [3].

A clear question raised by this methodology is whether the security of the cryptographic objects in an ideal world with a random oracle can be translated into security for the real-world implementation. In principle, this was answered negatively by Canetti, Goldreich and Halevi [7, who showed that there exist 
cryptographic schemes that are secure in the presence of a random oracle, but cannot be secure in the real world, regardless of the implementation of the random oracle. Their work left open the possibility that the random oracle methodology could still work for "natural" cryptographic practices. This was ruled out by Goldwasser and Kalai [1] for the Fiat-Shamir method [15], which uses a random oracle for obtaining digital signatures from identification schemes. The method was shown to lead to insecure signature schemes regardless of the possible implementation of the random oracle.

In the context of obfuscation, Lynn, Prabhakaran and Sahai [17] explored which circuits could be obfuscated in the (programmable) random oracle model, where the view generated by the black-box simulator is indistinguishable when taken over a randomly selected oracle. This work considers the same model. They used the random oracle $\mathcal{R}$ to obfuscate a point function $I_{p}$ (when $p$ is given to the obfuscator) using the value $\mathcal{R}(p)$. On input $x$ the obfuscated circuit outputs 1 if and only if $\mathcal{R}(x)=\mathcal{R}(p)$. The only information about $p$ in the obfuscated circuit is the value $\mathcal{R}(p)$, and this ensures that the obfuscation does not leak any non black-box information about $I_{p}$. They then proceeded to show how to obfuscate point functions with more general outputs (on input $x=p$ the function outputs some value, and otherwise it outputs $\perp$ ), multi-point functions and other more complex access control circuits. Narayanan and Shmatikov [16] gave a positive result for obfuscating databases in the random oracle model. In this work we explore whether indeed the random oracle model is a promising setting for further work on obfuscation.

\subsection{Organization}

We begin by presenting notation and formal definitions in Section 2, We compare our new definition of obfuscation with previous definitions in Section 3. In Section 4 we present impossibility results for statistically best-possible obfuscation, and for best-possible obfuscation in the random oracle model. We conclude with discussions and extensions in Section 5 .

\section{Definitions and Discussion}

\subsection{Notation and Preliminaries}

Notation. Let $[n]$ be the set $\{1,2, \ldots n\}$. For $x \in\{0,1\}^{n}$, where $x=x_{1} x_{2} \ldots x_{n}$, and an index subset $M \subseteq[n]$, where $M=\left\{i_{1}, i_{2}, \ldots i_{m}\right\}$, we use $\left.x\right|_{M}$ to denote the restriction of $x$ to the indices in $M$. I.e. $\left.x\right|_{M}=x_{i_{1}} x_{i_{2}} \ldots x_{i_{m}}$. For a (discrete) distribution $D$ over a set $X$ we denote by $x \sim D$ the experiment of selecting $x \in X$ by the distribution $D$. A function $f(n)$ is negligible if it smaller than any (inverse) polynomial: for any polynomial $p(n)$, there exists some $n_{0}$ such that for all $n \geq n_{0}$ we get that $f(n)<p(n)$.

Distributions, Ensembles and Indistinguishability. An ensemble $D=\left\{D_{n}\right\}_{n \in N}$ is a sequence of random variables, each ranging over $\{0,1\}^{\ell(n)}$, we consider only 
ensembles where $\ell(n)$ is polynomial in $n$ (we occasionally abuse notation and use $D$ in place of $D_{n}$ ). An ensemble $D$ is polynomial time constructible if there exists a probabilistic polynomial time Turing Machine (PPTM) $\mathcal{M}$ such that $D_{n}=\mathcal{M}\left(1^{n}\right)$.

Definition 1. The statistical distance between two distributions $X$ and $Y$ over $\{0,1\}^{\ell}$, which we denote by $\Delta(X, Y)$, is defined as:

$$
\Delta(X, Y)=\frac{1}{2} \sum_{\alpha \in\{0,1\}^{\ell}}|\operatorname{Pr}[X=\alpha]-\operatorname{Pr}[Y=\alpha]|
$$

Definition 2. Computational Indistinguishability (Goldwasser Micali [13], Yao [22]) Two probability ensembles $D$ and $F$ are computationally indistinguishable if for any PPTM $\mathcal{M}$, that receives $1^{n}$ and one sample $s$ from $D_{n}$ or $F_{n}$, and outputs 0 or 1 , there exists a negligible function neg, such that for all $n$ 's:

$$
\left|P r_{s \sim D_{n}}\left[\mathcal{M}\left(1^{n}, s\right)=1\right]-P r_{s \sim F_{n}}\left[\mathcal{M}\left(1^{n}, s\right)=1\right]\right| \leq \operatorname{neg}(n)
$$

The Random Oracle Model. In the random oracle model we assume that all parties (the circuits, obfuscator, adversary etc.) have access to a random oracle and can make oracle queries. All oracle queries are answered by a single function $\mathcal{R}$, that is selected uniformly and at random from the set of all functions. Specifically, for each input length $n, \mathcal{R}$ will be a function from $\{0,1\}^{n}$ to $\{0,1\}^{p(n)}$ for some polynomial $p$. For simplicity, we will assume throughout this work that for all $n$ 's the function $\mathcal{R}$ is a random permutation 11 on $\{0,1\}^{n}$. Circuits access the random oracle by making oracles queries using a special oracle gate. It is important that we assume that calls to these oracle gates are clearly visible when running the circuit.

\subsection{Definitions of Obfuscation}

In the subsequent definitions, we consider a family $\mathcal{C}$ of probabilistic polynomial size circuits to be obfuscated. For a length parameter $n$ let $\mathcal{C}_{n}$ be the circuits in $\mathcal{C}$ with input length $n$. The size of the circuits in $\mathcal{C}_{n}$ is polynomial in $n$. If the obfuscator $\mathcal{O}$ is a polynomial-size circuit, then we say it efficiently obfuscates the family $\mathcal{C}$, and that $\mathcal{C}$ is efficiently obfuscatable. Note that when considering obfuscation in the random oracle model, all circuits are allowed oracle access (including the circuits to be obfuscated), and all probabilities are taken over the selection of a random oracle. Whenever we refer to obfuscation, we will mean (efficient) black-box obfuscation unless explicitly noted otherwise.

Definition 3 (Black-Box Obfuscation [2]). An algorithm $\mathcal{O}$, which takes as input a circuit in $\mathcal{C}$ and outputs a new circuit, is said to be a black-box obfuscator for the family $\mathcal{C}$, if it has the following properties:

\footnotetext{
${ }^{1}$ Note that all our results hold for random function oracles (as long as the function's
} range is significantly larger than its domain, say at least twice as large.) 
- Preserving Functionality:

There exists a negligible function neg $(n)$, such that for any input length $n$, for any $C \in \mathcal{C}_{n}$ :

$$
\operatorname{Pr}\left[\exists x \in\{0,1\}^{n}: \mathcal{O}(C)(x) \neq C(x)\right] \leq \operatorname{neg}(n)
$$

The probability is over the random oracle and $\mathcal{O}$ 's coins.

- Polynomial Slowdown:

There exists a polynomial $p(n)$ such that for all but finitely many input lengths, for any $C \in \mathcal{C}_{n}$, the obfuscator $\mathcal{O}$ only enlarges $C$ by a factor of $p:|\mathcal{O}(C)| \leq p(|C|)$.

- Virtual Black-box:

For any polynomial size circuit adversary $\mathcal{A}$, there exists a polynomial size simulator circuit $\mathcal{S}$ and a negligible function neg $(n)$ such that for every input length $n$ and every $C \in \mathcal{C}_{n}$ :

$$
\left|\operatorname{Pr}[\mathcal{A}(\mathcal{O}(C))=1]-\operatorname{Pr}\left[\mid \mathcal{S}^{C}\left(1^{n}\right)=1\right]\right| \leq \operatorname{neg}(n)
$$

Where the probability is over the coins of the adversary, the simulator and the obfuscator. In the presence of a random oracle, the probability is also taken over the random oracle.

Definition 4 (Indistinguishability Obfuscation [2]). An algorithm $\mathcal{O}$, that takes as input a circuit in $\mathcal{C}$ and outputs a new circuit, is said to be a (computational/statistical/perfect) indistinguishability obfuscator for the family $\mathcal{C}$, if it has the preserving functionality and polynomial slowdown properties as above, and also has the following property (instead of the virtual black-box property).

- Computationally/Statistically/Perfectly Indistinguishable Obfuscation:

For all large enough input lengths, for any circuit $C_{1} \in C_{n}$ and for any circuit $C_{2} \in \mathcal{C}_{n}$ that computes the same function as $C_{1}$ and such that $\left|C_{1}\right|=\left|C_{2}\right|$, the two distributions $\mathcal{O}\left(\mathbf{C}_{\mathbf{1}}\right)$ and $\mathcal{O}\left(\mathbf{C}_{\mathbf{2}}\right)$ are (respectively) computationally/statistically/perfectly indistinguishable.

Definition 5 (Best-Possible Obfuscation). An algorithm $\mathcal{O}$, which takes as input a circuit in $\mathcal{C}$ and outputs a new circuit, is said to be a (computationally/statistically/perfectly) best-possible obfuscator for the family $\mathcal{C}$, if it has the preserving functionality and polynomial slowdown properties as above, and also has the following property (instead of the virtual black-box property).

\section{- Computational/Statistical/Perfect Best-Possible Obfuscation:}

For any polynomial size learner $\mathcal{L}$, there exists a polynomial size simulator $\mathcal{S}$ such that for every large enough input length $n$, for any circuit $C_{1} \in C_{n}$ and for any circuit $C_{2} \in \mathcal{C}_{n}$ that computes the same function as $C_{1}$ and such that $\left|C_{1}\right|=\left|C_{2}\right|$, the two distributions $\mathcal{L}\left(\mathcal{O}\left(\mathbf{C}_{\mathbf{1}}\right)\right.$ ) and $\mathcal{S}\left(\mathbf{C}_{\mathbf{2}}\right)$ are (respectively) computationally/statistically/perfectly indistinguishable. 
Informally, this definition guarantees that anything that can be learned efficiently from the obfuscated $\mathcal{O}\left(C_{1}\right)$, can also be extracted efficiently (simulated) from any program $C_{2}$ of similar size for the same function. Thus, any information that is exposed by $\mathcal{O}\left(C_{1}\right)$ is exposed by every other equivalent circuit of a similar size, and we conclude that $\mathcal{O}\left(C_{1}\right)$ is a better obfuscation than any of these other circuits.

When dealing with best-possible obfuscators, we often refer to the "empty" learner; this is the learner that simply outputs whatever obfuscation it gets as input. It is simple to see that if there exists an efficient simulator $\mathcal{M}$ for the "empty" learner, then there exists an efficient simulator $\mathcal{M}^{\prime}$ for every efficient learner $\mathcal{L}: \mathcal{M}^{\prime}$ on input $C_{2}$ simply computes $\mathcal{M}\left(C_{2}\right)$ and outputs the result of $\mathcal{L}\left(\mathcal{M}\left(C_{2}\right)\right)$. Thus, an equivalent definition to 'Best Possible' can do away with the leaner and only require the existence of an efficient simulator, i.e., a simulator $\mathcal{S}$ such that for circuits $C_{1}, C_{2}$ of identical size and identical functionality the distributions $\mathcal{O}\left(C_{1}\right)$ and $\mathcal{S}\left(C_{2}\right)$ are indistinguishable.

Note that when we refer to best-possible or indistinguishability obfuscators we always mean efficient and computational obfuscators unless we explicitly note otherwise. By perfect indistinguishability, we mean that the distributions are identical (statistical distance 0). For statistical indistinguishability, unless noted otherwise, we only assume that the distinguisher's advantage (the statistical distance) is smaller than a (specific) constant 2 This strengthens negative results.

\section{Comparison with Prior Definitions}

In this section we compare the new definition of best-possible obfuscation to the black-box and indistinguishability definitions proposed by Barak et al. [2].

\subsection{Best-Possible vs. Black-Box Obfuscation}

Best-possible obfuscation is a relaxed requirement that departs from the blackbox paradigm of previous work. We first observe that any black-box obfuscator is also a best-possible obfuscator.

Proposition 1. If $\mathcal{O}$ is an efficient black-box obfuscator for circuit family $\mathcal{C}$, then $\mathcal{O}$ is also an efficient (computationally) best-possible obfuscator for $\mathcal{C}$.

Proof. Assume for a contradiction that $\mathcal{O}$ is not a best-possible obfuscator for $C$. This implies that there is no best-possible simulator for the "empty" learner that just outputs the obfuscated circuit it gets. In particular, $\mathcal{O}$ itself is not a good simulator. Thus there exists a polynomial $p$ and a distinguisher $\mathcal{D}$, such

\footnotetext{
${ }^{2}$ The existence of an inefficient perfectly best-possible obfuscator, implies the existence of an efficient one that uses the simulator to obfuscate. A similar argument also applies to statistically best-possible obfuscation, unless the statistical distance guarantee is very weak.
} 
that for infinitely many input lengths $n$, there exist two circuits $C_{1}, C_{2} \in \mathcal{C}_{n}$, such that $\left|C_{1}\right|=\left|C_{2}\right|$ and $C_{1}$ and $C_{2}$ are equivalent, but:

$$
\left|\operatorname{Pr}\left[\mathcal{D}\left(\mathcal{O}\left(C_{1}\right)\right)=1\right]-\operatorname{Pr}\left[\mathcal{D}\left(\mathcal{O}\left(C_{2}\right)\right)=1\right]\right| \geq p(n)
$$

Now consider $\mathcal{D}$ as a predicate adversary for the black-box obfuscator $\mathcal{O}$. The black-box simulator $\mathcal{S}$ for $\mathcal{D}$ clearly behaves identically on $C_{1}$ and $C_{2}$ (because they have the same functionality), but $\mathcal{D}$ 's behavior on $\mathcal{O}\left(C_{1}\right)$ and $\mathcal{O}\left(C_{2}\right)$ is nonnegligibly different. Thus (for infinitely many input lengths) $\mathcal{S}$ is not a black-box simulator for $\mathcal{D}$, a contradiction.

Next, we provide a (weak) separation result. We exhibit a natural (low) complexity class, that of languages computable by polynomial size ordered binary decision diagrams (POBDDs), such that best-possible obfuscation within the class is achievable, but there are simple functionalities that are provably impossible to black-box obfuscate within the class.

Ordered Binary Decision Diagrams (OBDDs). The computational model of ordered binary decision diagrams was introduced by Bryant [5]. An ordered binary decision diagram is a rooted directed acyclic graph with a vertex set $V$ containing non-terminal vertices, each with two children, and terminal vertices (without children), each labeled 0 or 1 . Each edge $e$ in the graph is marked with an input literal $\ell_{e}$ (e.g. $\ell_{e}$ could be $x_{1}, \overline{x_{8}}$ etc.). For every non-terminal vertex, the labels of its (two) outgoing edges should be negations of each other (e.g. $x_{3}$ and $\overline{x_{3}}$ ). An input $x \in\{0,1\}^{n}$ is accepted by an OBDD if and only if after removing every edge $e$ for which $\ell_{e}=0$ there exists a path from the root node to a terminal node labeled by 1 . In addition, in an OBDD, on every path from the root vertex to a terminal vertex, the indices of the literals of edges on the path must be strictly increasing. We will focus on polynomial-size OBDDs, or POBDDs. We note that another way to view POBDDs is as logarithmic-space deterministic Turing Machines whose input tape head can only move in one direction (from the input's first bit to its last).

Bryant [5] showed that OBDDs have a simple canonical representation. For any function, there exists a unique smallest OBDD that is its canonical representation. Moreover, for polynomial-size OBDDs, this canonical representation is efficiently computable.

Note that we defined obfuscation for circuits, not OBDDs, but for every OBDD, there exists a boolean circuit (that computes the same functionality) from which it is easy to extract the OBDD. When we refer to obfuscating the family of OBDDs, we are implicitly referring to obfuscating the underlying family of circuits representing OBDDs.

We begin by observing that POBDDs can be perfectly best-possible obfuscated as POBDDs (namely the output of the obfuscator is a POBDD itself). This is a corollary of POBDDs having efficiently computable canonical representations. 
Proposition 2. There exists an efficient perfectly best-possible (and perfectly indistinguishable) obfuscator for POBBDs.

Proof. The best-possible obfuscator for a POBDD $P$ simply takes $P$, computes (efficiently) its canonical representation, and outputs that program as the bestpossible obfuscation. The canonical representation has the same functionality as $P$, is no larger than $P$, and (most significantly) is unique, depending only on the functionality of $P$. The simulator gets a POBDD $P^{\prime}$ and also efficiently computes its canonical representation. The canonical representations of $P$ and $P^{\prime}$ are identical if and only if $P$ and $P^{\prime}$ compute the same functionality. Thus the obfuscator is indeed a perfectly best-possible obfuscator for the family of POBDDs.

We next show that there exists a family of languages computable by POBDDs, that cannot be black-box obfuscated (efficiently or inefficiently) as POBDDs (i.e the resulting program itself being represented as a POBDD). This gives a (weak) separation between best-possible and black-box obfuscation. The weakness is that it remains possible that any input POBDD can be black-box obfuscated such that the output circuit is no longer a POBDD but is in some higher complexity class.

Proposition 3. There exists a family of languages computable by POBDDs, that cannot be black-box obfuscated as POBDDs.

Proof (Sketch). Intuitively, because POBDDs have a simple efficiently computable canonical representation, non black-box information can be extracted from a POBDD by reducing it to its "nice" canonical form, and then extracting information from this canonical form.

More formally, consider (for example) the simple family of point functions $\left\{I_{p}\right\}_{p \in\{0,1\}^{n}}$, where the function $I_{p}$ outputs 1 on input the point $p$ and 0 everywhere else. Note that point functions are computable by POBDDs. Now observe that any POBDD computing a point function for a point $p$ can be reduced to its canonical representation, from which $p$ is easily extracted. Thus for any supposed obfuscator that obfuscates point functions as POBDDs there exists an adversary that (for every point) can extract all the bits of the point from the "obfuscated" POBDD. Clearly, no black-box simulator can successfully extract even a single bit of the point for a non-negligible fraction of point functions. Thus there exists no black-box obfuscator that obfuscates POBDDS computing point functions as POBDDs.

We note that many other natural languages computable by POBDDs cannot be black-box obfuscated as POBDDs. Black-box obfuscation of POBDDs as more complex circuits remains an intriguing open question.

\subsection{Best-Possible vs. Indistinguishability Obfuscation}

As mentioned above, the notions of best-possible obfuscation and indistinguishability obfuscation are related, though the guarantees given by these two types 
of obfuscation are different. In this section we will show that any best-possible obfuscator is also an indistinguishability obfuscator. Furthermore, for efficient obfuscation, the two notions are equivalent. For inefficient obfuscation (which is still interesting), however, the notions are not equivalent unless the polynomial hierarchy collapses. In fact, inefficient indistinguishability obfuscators exist unconditionally (see [2]). On the other hand, building even inefficient bestpossible obfuscators remains an interesting open question. We begin by showing that best-possible obfuscation is in fact at least as strong as indistinguishability obfuscation.

Proposition 4. If $\mathcal{O}$ is a perfectly/statistically/computationally best-possible obfuscator for circuit family $\mathcal{C}$, then $\mathcal{O}$ is also a (respectively) perfect/statistical/ computational indistinguishability obfuscator for $\mathcal{C}$.

Proof (Sketch). To prove the claim, consider the "empty" learner $\mathcal{L}$ that just outputs whatever obfuscation it is given, and its simulator $\mathcal{S}$. Let $\delta$ be the computational or statistical distinguishability in the (computational or perfect/statistical) guarantee of the obfuscator. We get that for any two circuits $C_{1}$ and $C_{2}$ that are of the same size and compute the same functionality:

$$
\begin{aligned}
& \delta\left(\mathcal{L}\left(\mathcal{O}\left(C_{1}\right)\right), \mathcal{S}\left(C_{2}\right)\right)=\delta\left(\mathcal{O}\left(C_{1}\right), \mathcal{S}\left(C_{2}\right)\right) \leq \varepsilon \\
& \delta\left(\mathcal{L}\left(\mathcal{O}\left(C_{2}\right), \mathcal{S}\left(C_{2}\right)\right)\right)=\delta\left(\mathcal{O}\left(C_{2}\right), \mathcal{S}\left(C_{2}\right)\right) \leq \varepsilon
\end{aligned}
$$

Thus (since computational and statistical distinguishabilities are transitive):

$$
\delta\left(\mathcal{O}\left(C_{1}\right), \mathcal{O}\left(C_{2}\right)\right) \leq 2 \varepsilon
$$

Note that the perfect/statistical/computational guarantee is preserved.

As noted above, if we restrict our attention to efficient obfuscators, indistinguishability obfuscators are also best-possible obfuscators.

Proposition 5. If $\mathcal{O}$ is an efficient perfect/statistical/computational indistinguishability obfuscator for a circuit family $\mathcal{C}$, then $\mathcal{O}$ is also an efficient (respectively) perfectly/statistically/computationally best-possible obfuscator for $\mathcal{C}$.

Proof. Let $\mathcal{O}$ be an efficient indistinguishability obfuscator. Then for any learner $\mathcal{L}$, let $\mathcal{S}$ be the (efficient) simulator that gets a circuit $C_{2}$, runs $\mathcal{O}\left(C_{2}\right)$, and then activates $\mathcal{L}$ on $\mathcal{O}\left(C_{2}\right)$. We get that if $\mathcal{O}$ is a perfect/statistical/computational indistinguishability obfuscator, then for any two circuits $C_{1}$ and $C_{2}$ that are of the same size and compute the same functions, the two distributions $\mathcal{L}\left(\mathcal{O}\left(C_{1}\right)\right)$ and $\mathcal{S}\left(C_{2}\right)=\mathcal{L}\left(\mathcal{O}\left(C_{2}\right)\right)$ are perfectly/statistically/computationally indistinguishable (because $\mathcal{O}$ is an indistinguishability obfuscator). Thus $\mathcal{O}$ is also an efficient best-possible obfuscator.

Note that the efficiency of the indistinguishability obfuscator is essential to guarantee the efficiency of the simulator, without which the obfuscator does not meet the best-possible definition. 
It is important to note that there is no reason to believe that the two notions of obfuscation are equivalent for inefficient obfuscation. In fact, whereas [2] design exponential-time indistinguishability obfuscators, there is no known construction for inefficient best-possible obfuscators. We believe that even constructing inefficient best-possible obfuscators is interesting.

We end this subsection by observing that if $P=N P$ then it is not hard to construct efficient perfect best-possible obfuscators (and indistinguishability obfuscators) for every polynomial-size circuit. In fact this complexity assumption is almost tight. We will show in Theorem 1, that if statistically best-possible obfuscators can be built even for very simple circuits, then the polynomial hierarchy collapses to its second level.

Proposition 6. If $P=N P$ then the family of polynomial-sized circuits can be efficiently perfectly best-possible obfuscated.

Proof. Assume $P=N P$. For any circuit $C$, it is possible to efficiently extract the smallest lexicographically first circuit $C_{m i n}$ that is equivalent to $C$ (this problem is solvable using a language in the second level of the polynomial hierarchy). As Barak et al. 2] note, such an extraction procedure is a perfectly indistinguishable obfuscation of $C$, and thus there exists an efficient perfect indistinguishability obfuscator for the family of polynomial-size circuits. By Proposition 5 it is also an efficient perfect best-possible obfuscator for the family of polynomial-size circuits. Note that even if $P \neq N P$ then we get an (inefficient) indistinguishability obfuscator. It remains, however, unclear whether we can get an inefficient bestpossible obfuscator, as the (always efficient!) simulator can no longer run the "circuit minimization" procedure.

\section{Impossibility Results for Best-Possible Obfuscation}

\subsection{Statistically Best-Possible Obfuscation}

In this section we present a hardness result for statistically best-possible obfuscation. In Section 3 it was shown that if $P=N P$ then every polynomial-sized circuit can be perfectly best-possible obfuscated, thus we cannot hope for an unconditional impossibility result. We show that the condition $P=N P$ is (nearly) tight, and in fact the existence of statistically best-possible obfuscators even for the class of languages recognizable by 3-CNF circuits (a sub-class of $A C^{0}$ ) implies that the polynomial hierarchy collapses to its second level. This result shows the impossibility of statistically best-possible obfuscation for any class that contains 3-CNF formulas (and in particular also for the class of general polynomial sized circuits).

Theorem 1. If the family of 3-CNF formulas can be statistically best-possible obfuscated (not necessarily efficiently), then the polynomial hierarchy collapses to its second level. 
Proof (Intuition). We begin by considering the case that the family of 3-CNF formulas can be perfectly best-possible obfuscated (not necessarily efficiently) while perfectly preserving functionality (i.e. the obfuscated circuit never errs). We can use the Simulator $\mathcal{S}$ for the "empty" learner, to construct an NP proof for Co-SAT (a Co-NP -complete problem). To see this, consider an input 3-CNF formula $\varphi$ of size $|\varphi|$. We would like to find a witness for non-satisfiability of $\varphi$. Towards this end, we first construct an unsatisfiable formula $\psi$ of size $|\varphi|$. A witness for the non-satisfiability of $\varphi$ is a pair of random strings $\left(r, r^{\prime}\right)$ such that the output of the simulator $\mathcal{S}$ on $\varphi$ with randomness $r$ is equal to its output on $\psi$ with randomness $r^{\prime}$. This proof system is indeed in $N P$ :

- Efficiently Verifiable. The simulator is efficient, and thus the witness is efficiently verifiable.

- Complete. If $\varphi$ is unsatisfiable, then $\varphi$ and $\psi$ compute the same function (the constant 0 function) and are of the same size. We know that $\mathcal{O}$ is a perfect best-possible obfuscator and thus the distributions $\mathcal{O}(\varphi), \mathcal{S}(\varphi)$, $\mathcal{S}(\psi), \mathcal{O}(\psi)$ are all identical. This implies that there must exist $\left(r, r^{\prime}\right)$ such that $\mathcal{S}(\varphi, r)=\mathcal{S}\left(\psi, r^{\prime}\right)$.

- Sound. If $\varphi$ is satisfiable, then because the obfuscator perfectly preserves functionality, the distributions $\mathcal{O}(\varphi), \mathcal{O}(\psi)$ are disjoint (they are distributions of circuits with different functionalities). Thus the distributions $\mathcal{S}(\varphi)$, $\mathcal{S}(\psi)$ of the (perfect) simulator's output are also disjoint, and there exist no $\left(r, r^{\prime}\right)$ such that $\mathcal{S}(\varphi, r)=\mathcal{S}\left(\psi, r^{\prime}\right)$.

The full proof for the case of statistically best-possible obfuscation follows along similar lines, giving a reduction from a Co-NP -complete problem (circuit equivalence) to a problem in $A M \sqrt[3]{3}$ By the results of Fortnow [10, Aliello and Håstad [1, and Boppana, Håstad and Zachos 4] (see also Feigenbaum and Fortnow [14]), this collapses the polynomial hierarchy to its second level. The full proof is omitted from this extended abstract.

Proposition 2 and Theorem 1 give examples of circuit classes can and cannot be statistically best-possible obfuscated. The proofs give characterizations of circuit classes that can be statistically best-possible obfuscated. A sufficient condition for statistically best-possible obfuscation of a class of circuits is having an efficiently computable canonical representation, a necessary condition is having a statistical zero knowledge proof for the equivalence problem.

Finally, a corollary of this theorem is that the same class of 3-CNF formulas cannot be statistically indistinguishability obfuscated in polynomial time unless the polynomial hierarchy collapses. This is the first impossibility result for indistinguishability obfuscation in the standard model.

Corollary 1. If the family of 3-CNF formulas can be efficiently statistically indistinguishability obfuscated, then the polynomial hierarchy collapses to its second level.

${ }^{3}$ Actually, this is a problem in statistical zero knowledge: the complement of the Statistical Difference Problem, introduced by Sahai and Vadhan 20. 
Proof. By Proposition 5, if there exists an efficient statistical indistinguishability obfuscator for the family of 3 -CNFs, then there also exists an efficient statistically best-possible obfuscator for the same family. This, in turn, implies (by Theorem 1) that the polynomial hierarchy collapses to its second level.

\subsection{Computationally Best-Possible Obfuscation}

In this section we present an impossibility result for (efficient) computationally best-possible obfuscation in the (programmable) random oracle model. We show how to use a random oracle to build circuits for point functions that cannot be best-possible obfuscated. We note that the use of the random oracle both strengthens and weakens this result. The result is strengthened because a random oracle could conceivably help obfuscation ( la [17]), but weakened because the random oracle is used to build a circuit that cannot be obfuscated. Moreover, in the proof we need to assume that a distinguisher can see the obfuscated circuit's oracle calls and that it can access the random oracle itself. It is still possible that circuits that do not use the random oracle can be best-possible obfuscated.

We show that a specific family of circuits for computing point functions cannot be obfuscated in the presence of a random oracle $\mathcal{R}$. A point function $I_{p}$ is the function that outputs 1 on input $p$ and 0 on all other inputs. We begin by presenting the family of point function circuits for which we will show impossibility of obfuscation.

Definition 6 (The circuit family $\left\{C_{p}^{M}\right\}$ ). For any input length $n$, the family of circuits $\left\{C_{p}^{M}\right\}_{n}$ defines a set of circuits on inputs of length $n$. Each circuit $C_{p}^{M}$ computes the point function $I_{p}$ on the point $p \in\{0,1\}^{n}$, and is defined by the point $p$ and an index subset $M \subseteq[n]$ (all index subsets in this section are of size $\left.\frac{n}{2}\right)$. The information that the circuit $C_{p}^{M}$ gives about $p$ is:

- The index subset $M$ is included in $C_{p}^{M}$ "in the clear".

- The bits of $p$ that aren't in the index subset $M\left(\left.p\right|_{[n]-M}\right)$ are also given in the clear.

- The bits of $p$ that are in $M\left(\left.p\right|_{M}\right)$ are "hidden", the only information given about them is $\mathcal{R}\left(\left.p\right|_{M}\right)$.

For an input $x$, to compute the point function $I_{p}$, the circuit $C_{p}^{M}$ outputs 1 if and only if $x$ is equal to $p$ in the indices that aren't in $M\left(\left.x\right|_{[n]-M}=\left.p\right|_{[n]-M}\right)$, and the random oracle gives the same values on $x$ and $p$ restricted to $M\left(\mathcal{R}\left(\left.x\right|_{M}\right)=\right.$ $\left.\mathcal{R}\left(\left.p\right|_{M}\right)\right)$. Thus $C_{p}^{M}(x)=1$ if and only if $x=p$, otherwise the circuit outputs 0 .

We also take the family $\left\{I_{p}\right\}$ to be the family of point function circuits that contain their point in the clear, and are padded to be of the same size as the circuits $\left\{C_{p}^{M}\right\}$ on each input length. We claim that the family of point function circuits $\left\{C_{p}^{M}\right\} \cup\left\{I_{p}\right\}$ cannot be best-possible obfuscated.

Theorem 2. The circuit family $\left\{C_{p}^{M}\right\} \cup\left\{I_{p}\right\}$ cannot be efficiently computationally best-possible obfuscated. 
Proof (Proof Intuition). Observe that any obfuscator $\mathcal{O}$ must preserve the functionality of a circuit $C_{p}^{M}$. Furthermore, the only information the obfuscator has about the indices of the point $p$ that are in the subset $M$ is the value $\mathcal{R}\left(\left.p\right|_{M}\right)$. To preserve functionality, for any input $x$, the obfuscated circuit $\mathcal{O}\left(C_{p}^{M}\right)$ needs to find out whether $x=p$. Now because the only information available to the obfuscator and the obfuscated circuit about $\left.p\right|_{M}$ is the value $\mathcal{R}\left(\left.p\right|_{M}\right)$, for most inputs $x$, the obfuscated circuit must ask the random oracle for the value $\mathcal{R}\left(\left.x\right|_{M}\right)$. Thus for many $x$ 's one of the (polynomially many) oracle calls of $\mathcal{O}\left(C_{p}^{M}\right)$ should be to $\mathcal{R}\left(\left.x\right|_{M}\right)$.

In the proof we construct a distinguisher between obfuscated circuits and the output of the "empty" learner's simulator. For an index subset $T \subseteq[n]$ and input $x \in\{0,1\}^{n}$, we examine the distinguisher $\mathcal{D}_{T, x}$ that activates the obfuscated circuit it was given, $\mathcal{O}\left(C_{p}^{M}\right)$ (for some index subset $M$ and point $p$ ), on the input $x$, and tries to guess whether $T$ was the subset used in the underlying circuit that was obfuscated (i.e. whether $M=T$ ). To do this, the distinguisher runs $\mathcal{O}\left(C_{p}^{M}\right)$ on the input $x$ and outputs 1 if and only if the obfuscated circuit queried the random oracle on the input $\left.x\right|_{T}$. Recall that we concluded above that if $M=T$, then we expect the obfuscated circuit to query the random oracle on the input $\left.x\right|_{T}$. Thus, when the distinguisher $\mathcal{D}_{T, x}$ gets $\mathcal{O}\left(C_{p}^{M}\right)(x)$, it has an advantage in deciding whether $M=T$ or not. This advantage disappears when the distinguisher is activated on the output of a simulator that was given the circuit $I_{p}$ : the simulator was given no information about $M$, so its output cannot help the distinguisher determine whether or not $M=T$. The full proof is omitted from this extended abstract.

The family of circuits that we show cannot be obfuscated is a family that computes point functions. This may seem contradictory, as Lynn, Prabhakaran and Sahai [17] showed that a class of circuits computing point functions can be obfuscated in the random oracle model. The source of this disparity is that they (as well as all other known positive results on obfuscating point functions) only consider obfuscators that get the point in the clear, whereas the family of point function circuits that we present $\left(\left\{C_{p}^{M}\right\}\right)$ hides information about the point. Malkin [18, was the first to ask whether any point function implementation can be black-box obfuscated.

Thus Theorem 2 shows impossibility for simpler and more natural functionalities than those considered in previous results, but does so using circuits with random oracle gates.

Extensions. We note that this impossibility result applies also to black-box obfuscation (the proof is omitted from this extended abstract, but note that the distinguisher in the theorem can be viewed as a predicate adversary). One possible objection to this impossibility result, is that the information revealed by obfuscation of circuits in the family $\left\{C_{p}^{M}\right\}$ (namely the subset $M$ ) is not necessarily information related to the point $p$. We note, however, that unless an obfuscator guarantees that no non black-box information is revealed by the 
obfuscation, for circuits for which the point $p$ is related to the subset $M$, the obfuscated circuit may leak non black-box information about the point $p$.

Implications for a world without random oracles. We conclude with a discussion of the ways in which our proof uses the random oracle model, and how one could hope to remove this assumption. Our construction uses the random oracle $\mathcal{R}$ in two ways. First, $\mathcal{R}$ is used to hide information about $p$ in the circuit family $\left\{C_{p}^{M}\right\}$. Essentially, we use $\mathcal{R}$ to obfuscate a point function (where the point is $\left.\left.p\right|_{M}\right)$. Intuitively, since we know how to (black-box) obfuscate point functions without using random oracles, we could use (strong) cryptographic assumptions in place of the random oracle for this.

The second place in our proof where we use the properties of random oracles is when we assume a distinguisher can see the points on which the obfuscated circuit queries the random oracle. If we want to get rid of the random oracles, this is a more troubling assumption. The issue is that even if we could use some other method to hide information about the point $p$ in the standard model, there is no reason to assume we could identify any internal computation of the obfuscated circuit. For example, consider using Canetti's point function obfuscation and giving the obfuscator a circuit $C$ that hides some information on $p$ by exposing only $\left(r, r^{\left.p\right|_{M}}\right)$. Even if on any input $x$ the obfuscated circuit always computes $\left(r, r^{\left.x\right|_{M}}\right)$, there is no guarantee that a distinguisher can identify these computations! Thus $\mathcal{O}(C)$ may not expose any information on $M$. We note, however, that to prove that an obfuscator can obfuscate any circuit computing a point function, one would have to construct an obfuscator that indeed hides internal computations. Thus it seems that even for achieving the (seemingly modest) goal of best-possible obfuscation for polynomial-size point-function circuits, one would have to present a method for hiding complex internal computations of a circuit. Such a method, in and of itself, would likely have interesting implications.

\section{Concluding Remarks and Discussions}

We conclude with a discussion of best-possible obfuscation and issues raised in this work.

Input/Output Representation. Several of our results highlight the issue of the representation of an obfuscator's input and output. At times (in Section 3) we restrict the representation of both the obfuscator's input and output functionality to be "simple" circuits representing POBDDs. At other times (in the proof of Theorem 2), we construct complex circuits that hide information about their functionality from the obfuscators. In general, restricting the input representation makes the task of obfuscation easier (see discussion in section 4.2), whereas restricting the output representation makes the task of obfuscation harder, and we use this in Proposition 3 to show that point functions cannot be black-box obfuscated as POBDDs. Previous positive results on obfuscation considered obfuscators that get a particular representation of the functionality (e.g. the point 
$p$ for the point function $I_{p}$ ). Future work on black-box (and non black-box) obfuscation should consider the question of which representations of the desired functionality are obfuscated.

This issue was also raised by Malkin [18, who asked whether any point function implementation can be black-box obfuscated in the standard model. An relaxed (but related f formulation of this question is whether the family of polynomial-size circuits computing point functions can be best-possible obfuscated. The proof of Theorem 2 answers this question negatively in the presence of random oracles, but either an impossibility proof or a provably secure obfuscator would likely have interesting consequences.

Circuit Sizes. In our definition of best-possible obfuscation (Definition [5) we compare the obfuscated circuit $\mathcal{O}\left(C_{1}\right)$ with circuits $C_{2}$ of the same size as $C_{1}$ (and computing the same functionality). This definition requires that the obfuscation of $C_{1}$ leak as little information as any equivalent circuit of a specific (polynomially) smaller size. We could make stronger requirements, such as leaking less information than an equivalent circuit $C_{2}$ that is as large as $\mathcal{O}\left(C_{1}\right)$, twice as large as $C_{1}$, etc. (all results would still hold). In general, the larger the circuit used as a benchmark $\left(C_{2}\right)$, the stronger the definition. The important point is guaranteeing that $\mathcal{O}\left(C_{1}\right)$ leaks as little information as any other functionally equivalent circuit of a related size.

Auxiliary Input. Goldwasser and Kalai [12] augment the virtual black-box requirement of obfuscation to hold in the presence of auxiliary input. They note that this is an important requirement for any obfuscation that is used in practice, as auxiliary input comes into play in the real world. Following this argument, we could extend the best-possible obfuscation requirement to hold in the presence of auxiliary input. This is a strengthening of the definition, and thus all negative results clearly still hold. The positive result of Proposition 2 (obfuscating POBDDs) also holds even in the presence of (dependent) auxiliary input.

Weaker Variants. In light of the negative results of Theorems 1 and 2 it is interesting to consider weaker variants of best-possible obfuscation (Definition 5). While the variants below lose some of the appealing intuitive "garbling" guarantee of Definition 5, meeting any of them would all give at least some indication that the obfuscator truly garbles circuits.

- Hiding Less Information. One natural approach is to follow in the footsteps of Barak et al. [2, and consider best-possible predicate obfuscators: an obfuscation is predicate best-possible if any predicate of the original circuit that can be learned from the obfuscation, could also be learned from any other circuit of a similar size computing the same functionality. While this definition is weaker than computationally best-possible obfuscation, the proof of

\footnotetext{
${ }^{4}$ This formulation is equivalent to the original question raised by Malkin under the assumption that point functions can indeed be obfuscated when the point is given in the clear. In this case, a best-possible obfuscation leaks as little information as the black-box obfuscated point function circuits, and is thus also a black-box obfuscation.
} 
Theorem 2 rules out even general-purpose predicate best-possible obfuscation in the random oracle model (and gives some intuition that this type of obfuscation would be hard to achieve int he standard model).

- Weaker Indistinguishability. Canetti 6] and Wee 21 relax the virtual blackbox requirement, requiring only polynomially small indistinguishability between the output of an adversary and its simulator. Moreover, they allow the simulator's size to depend (polynomially) on this indistinguishability parameter. We note that negative results in this work (Theorems 1 and 2) hold even if we require only polynomially small indistinguishability and allow the simulator's size to depend (polynomially) on the indistinguishability parameter.

- Weaker Functionality. Definition 5 requires that with all but negligible probability, the obfuscated circuit perfectly preserves the functionality of the original circuit. We could relax this, and require only that for every input, with all but a small constant error probability, the obfuscated circuit outputs the same output as the original circuit. Our negative results apply even under this weakened preserving functionality requirement. The positive result on best-possible obfuscation of POBDDs (Proposition 21) gives an obfuscator that perfectly preserves the functionality of the circuit it obfuscates.

\section{Acknowledgements}

We thank Yael Tauman Kalai and Tali Kaufman for helpful and enjoyable discussions. Thanks also to the anonymous reviewers for their insightful comments which much improved (or so we hope) the presentation.

\section{References}

1. William Aiello, Johan Håstad. Statistical Zero-Knowledge Languages can be Recognized in Two Rounds. Journal of Computer and System Sciences 42(3): 327-345 (1991)

2. Boaz Barak, Oded Goldreich, Russell Impagliazzo, Steven Rudich, Amit Sahai, Salil P. Vadhan, Ke Yang. On the (Im)possibility of Obfuscating Programs. CRYPTO 2001: $1-18$

3. Mihir Bellare, Phillip Rogaway. Random Oracles are Practical: A Paradigm for Designing Efficient Protocols. ACM Conference on Computer and Communications Security 1993: 62-73

4. Ravi B. Boppana, Johan Håstad, Stathis Zachos. Does co-NP Have Short Interactive Proofs? Information Processing Letters 25(2): 127-132 (1987)

5. Randal E. Bryant. Graph-Based Algorithms for Boolean Function Manipulation. IEEE Transactions on Computers, C(35), No. 8, August, 1986: 677-691

6. Ran Canetti. Towards Realizing Random Oracles: Hash Functions That Hide All Partial Information. CRYPTO 1997: 455-469

7. Ran Canetti, Oded Goldreich, Shai Halevi. The random oracle methodology, revisited. Journal of the ACM 51(4): 557-594 (2004)

8. Ran Canetti, Daniele Micciancio, Omer Reingold. Perfectly One-Way Probabilistic Hash Functions (Preliminary Version). STOC 1998: 131-140 
9. Yevgeniy Dodis, Adam Smith. Correcting errors without leaking partial information. STOC 2005: 654-663

10. Lance Fortnow. The complexity of perfect zero-knowledge. In S. Micali, editor, Advances in Computing Research, 5: 327-343. JAI Press, Greenwich, 1989

11. Shafi Goldwasser, Yael Tauman Kalai. On the (In)security of the Fiat-Shamir Paradigm. FOCS 2003: 102-113

12. Shafi Goldwasser, Yael Tauman Kalai. On the Impossibility of Obfuscation with Auxiliary Input. FOCS 2005: 553-562

13. Shafi Goldwasser and Silvio Micali. Probabilistic Encryption and How to Play Mental Poker Keeping Secret All Partial Information. STOC 1982: 365-377

14. Joan Feigenbaum, Lance Fortnow. Random-Self-Reducibility of Complete Sets. SIAM Journal on Computing 22(5): 994-1005 (1993)

15. Amos Fiat, Adi Shamir. How to Prove Yourself: Practical Solutions to Identification and Signature Problems. CRYPTO 1986: 186-194

16. Arvind Narayanan, Vitaly Shmatikov. Obfuscated databases and group privacy. ACM Conference on Computer and Communications Security 2005: 102-111

17. Ben Lynn, Manoj Prabhakaran, Amit Sahai. Positive Results and Techniques for Obfuscation. EUROCRYPT 2004: 20-39

18. Tal Malkin. Personal Communication (2006).

19. Tatsuaki Okamoto. On Relationships between Statistical Zero-Knowledge Proofs. Journal of Computer and System Sciences 60(1): 47-108 (2000)

20. Amit Sahai, Salil P. Vadhan. A complete problem for statistical zero knowledge. Journal of the ACM 50(2): 196-249 (2003)

21. Hoeteck Wee. On obfuscating point functions. STOC 2005: 523-532

22. Andrew Chi-Chih Yao. Theory and Applications of Trapdoor Functions (Extended Abstract). FOCS 1982: 80-91 